$41-47$

\title{
Dropped head syndrome: diagnosis and management
}

Authors Allan R Martin, Rajesh Reddy, Michael G Fehlings

Institution Division of Neurosurgery and Krembil Neuroscience-Spinal Program, Toronto Western Hospital, University of Toronto, Toronto, Ontario, Canada

\section{ABSTRACT}

Dropped head syndrome (DHS) is a relatively rare condition, with a broad differential diagnosis. This deformity has significant implications on the health and quality of life of affected individuals. While surgery seems to be an obvious therapeutic option, there is a paucity of information on surgical intervention with no clear consensus on an optimal approach or timing.

We present a case of DHS in a young woman to illustrate this condition, and review the current literature. Although at present the only definitive solution for correction and stabilization of DHS is surgical intervention involving multilevel instrumented fixation and fusion, this condition requires a persistent medical workup and treatment of reversible causes before surgical intervention is contemplated. 


\section{BACKGROUND}

Dropped head syndrome (DHS) is characterized by severe kyphotic deformity of the cervico-thoracic spine. It is a relatively rare condition with a broad differential diagnosis [1]. The conditions linked with DHS can be categorized into neurological, neuromuscular, muscular, and other causes (Table 1). This deformity has significant implications on the quality of life of affected individuals, resulting in considerable restrictions to ambulation, activities of daily living, and social interactions. While surgery seems to be an obvious therapeutic option, there is a paucity of information on surgical intervention with no clear consensus on an optimal approach or timing.

Table 1 Summary of conditions associated with dropped head deformity.

\begin{tabular}{|c|c|c|}
\hline \multicolumn{2}{|c|}{ Type of condition } & Specific diagnosis \\
\hline \multirow{8}{*}{\multicolumn{2}{|c|}{ Neurological }} & Amyotrophic lateral sclerosis $[9,10]$ \\
\hline & & Parkinson disease [11] \\
\hline & & Multiple system atrophy [12] \\
\hline & & Cervical dystonia [13] \\
\hline & & Postpolio syndrome [14] \\
\hline & & Cervical myelopathy [7] \\
\hline & & Chronic inflammatory polyneuropathy [15] \\
\hline & & Tardive dyskinesia [16] \\
\hline \multirow{2}{*}{\multicolumn{2}{|c|}{ Neuromuscular }} & Myasthenia gravis [17] \\
\hline & & Lambert-Eaton myasthenic syndrome [18] \\
\hline \multirow[t]{16}{*}{ Muscular } & \multirow[t]{3}{*}{$\begin{array}{l}\text { Primary, } \\
\text { inflammatory }\end{array}$} & Polymyositis [19] \\
\hline & & Scleromyositis [20] \\
\hline & & Isolated inflammatory axial myopathy [21] \\
\hline & \multirow[t]{6}{*}{$\begin{array}{l}\text { Primary, } \\
\text { noninflammatory }\end{array}$} & Nemaline myopathy [12] \\
\hline & & Inclusion body myopathy [22] \\
\hline & & Mitochondrial myopathy [23] \\
\hline & & Congenital myopathy \\
\hline & & Fascioscapulohumeral dystrophy \\
\hline & & Isolated neck extensor myopathy [22] \\
\hline & \multirow[t]{7}{*}{ Secondary } & $\begin{array}{l}\text { Postradiation neck extensor myopathy } \\
\text { Anterior scar contracture [24] }\end{array}$ \\
\hline & & Postbotulinum toxin injection [13] \\
\hline & & Cushing syndrome [25] \\
\hline & & Carnitine deficiency [26] \\
\hline & & Hypothyroidism [27] \\
\hline & & Hypokalemia [28] \\
\hline & & Hyperparathyroidism $[29,30]$ \\
\hline \multirow{2}{*}{\multicolumn{2}{|c|}{ Other }} & Malignancy [31] \\
\hline & & Postsurgical [8] \\
\hline
\end{tabular}

\section{CASE REPORT}

A 38-year-old woman presented to the senior author's spinal cord clinic with a history of severe chronic cervical pain and a recent history of cervical deformity and gait abnormalities. She gave a decade long history of gradually worsening intense tightness around the neck and muscular contractions. Her neck deformity had been slowly progressive over the last 3 to 4 years but had worsened in recent months. She also described trouble ambulating, primarily due to difficulty holding up her head. However, she testified no motor or sensory dysfunction in any extremity.

On examination she was found to have pronounced cervical kyphosis with hypertrophic neck extensors. The deformity was completely reducible by the examiner. Active cervical flexion and extension were reduced to $50 \%$ of normal and painful at extremes. Muscle bulk, tone, and power were normal in all four limbs. She had mild global hyperreflexia and positive Hoffmann reflexes bilaterally, but downward Babinski reflexes bilaterally. Tests of sensation were normal.

X-ray of cervical spine (Fig 1) indicated marked cervical kyphosis centered on the mid-cervical spine, and evidence of spondylosis. Magnetic resonance imaging (MRI) (Fig 2) showed multilevel cervical spondylosis with cerebrospinal fluid effacement at the mid-cervical levels but no evidence of cord signal change. A preliminary diagnosis of cervical dystonia causing DHS with subtle signs of myelopathy was considered, and further investigations to determine the primary pathology were organized.

Initial electrodiagnostic findings were nonspecific. A muscle biopsy from the trapezius showed no evidence of inflammatory myopathy and nonspecific atrophic changes with fibrosis which were considered nondiagnostic.

Over 2 years she became progressively disabled with decline in her functional state such that she could only ambulate short distances while holding her head up with both hands. Clinical examination showed an increase in hyperreflexia of the left arm but was otherwise unchanged. Repeated MRI (Fig 3) showed distinct T2 hyperintensity of the cord at $\mathrm{C} 3-\mathrm{C} 4$ and atrophy with fat infiltration of the paraspinal muscles in the lower cervical spine. Repeated electrodiagnostic assessment showed fibrillation and small amplitude polyphasic potentials in the paraspinal muscles suggesting myopathy as the cause of DHS.

The patient consented to surgical intervention to achieve the goals of decompression of neural structures, and cor- 
rection of the cervical deformity with stabilization. She underwent a C2 to $\mathrm{T} 1$ decompressive laminectomy, and occiput to T6 posterior instrumented fixation and fusion. Good reduction of the cervical deformity with neutral head alignment was achieved via direct visualization and image intensification. The surgical procedure was uncomplicated, with motor and sensory-evoked potentials maintained throughout. Intraoperative electromyographic recordings showed transient-spiking activity during the procedure but this resolved fast.

Fig 1 X-ray of cervical spine demonstrated marked kyphotic deformity and evidence of spondylosis.

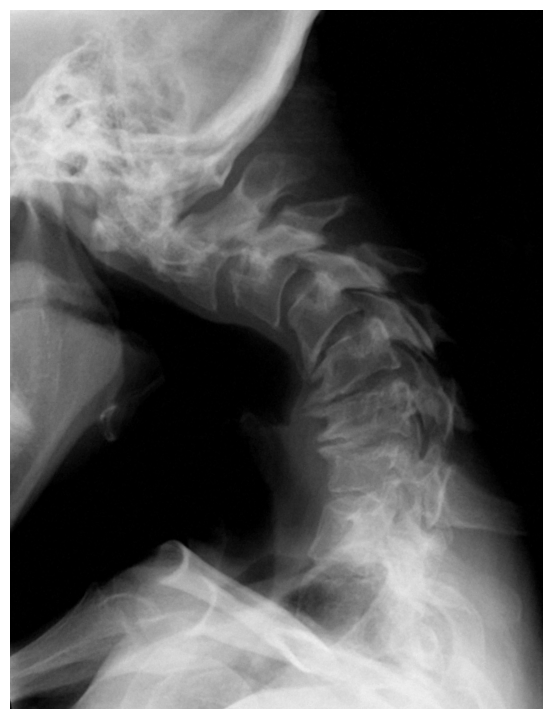

Fig 3 Magnetic resonance imaging (mid-sagittal T2-weighted image) from 2010. Subtle progression of spondylosis, now with distinct T2 hyperintensity of the cord at C3-C4. Evidence also of fat infiltration of the paraspinal muscles in the lower cervical spine region.

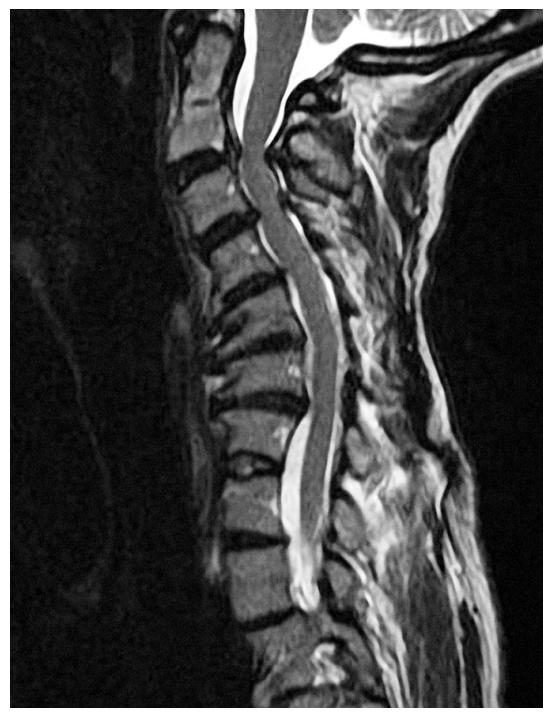

Postoperatively she recovered well with no neurological deficits. Computed tomography confirmed good positioning of all hardware, and anatomical alignment with increased space in the spinal canal. She was managed for 8 weeks postoperatively in a cervico-thoracic orthosis. She regained full mobility, and at 8 months follow-up she reported increased energy and a significantly improved ability to carry out daily activities. Imaging at follow-up shows the correction and stabilization of the deformity (Fig 4).

Fig 2 Magnetic resonance imaging (mid-sagittal T2-weighted image) from 2007, when the patient initially presented. Evidence of spondylosis with cerebrospinal fluid effacement at the mid-cervical levels, although no evidence of cord signal change.

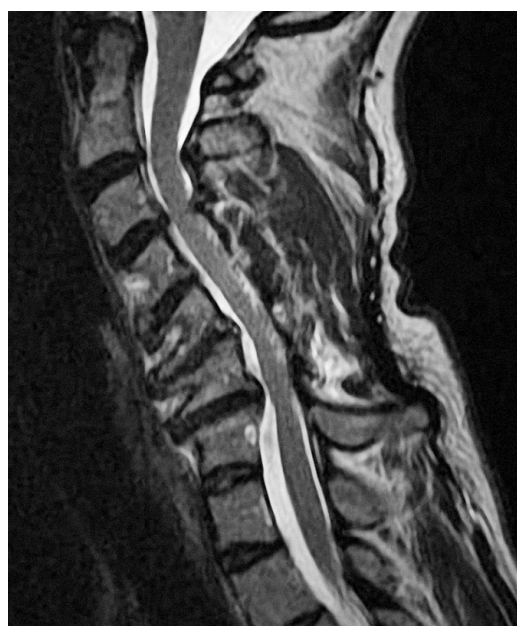

Fig 4 X-ray of cervical spine at follow-up. Extent of correction of kyphotic deformity is shown.

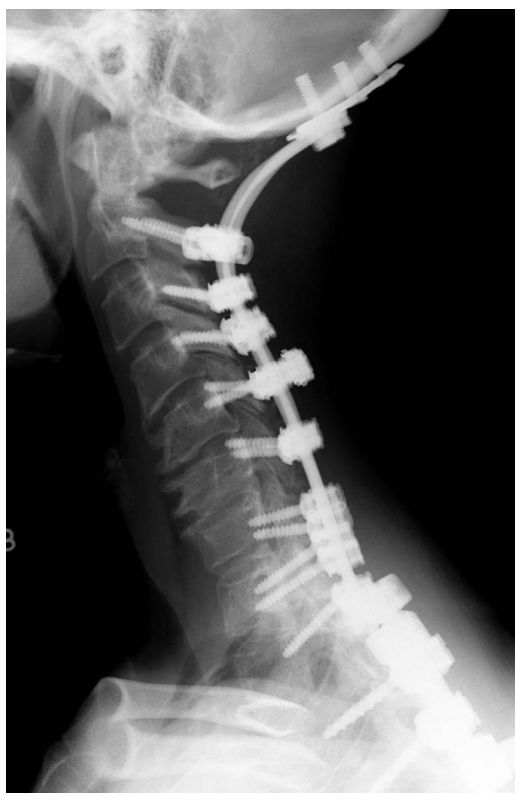




\section{DISCUSSION}

Dropped head syndrome is a complex and challenging condition. Optimal management of a patient with DHS requires a comprehensive multidisciplinary workup to establish the primary cause before surgical decision making. Many of the underlying conditions are medically treatable and in some cases the extent of the deformity may be decreased to an acceptable level without invasive intervention. In particular, Parkinson disease, inflammatory myositis, and secondary myopathies due to metabolic or endocrine dysfunction are likely to show substantial improvement when treated with appropriate medications. Other etiologies that cause focal structural changes are poorly suited to medical treatment, such as neck radiation therapy causing extensor myopathy and anterior scar contracture.

When investigations including electromyographic studies and muscle biopsy show a myopathic cause of DHS that is noninflammatory and isolated to the neck extensors, a diagnosis of isolated neck extensor myopathy can be made. While isolated neck extensor myopathy and some other causes of DHS may not be medically treatable, benefit may be gained with physiotherapy, massage, and acupuncture to maximize function and slow progression of symptoms. However, the natural history of this condition usually involves progression to a complete inability to lift the head in a seated or standing position, and this severe kyphosis can lead to secondary degenerative changes including vertebral compression and anterior muscle contraction. This development can later lead to cervical myelopathy and neurological decline. Thus, when medical and physical treatment options have been exhausted, mechanical correction of DHS may be needed to maintain a reasonable quality of life for the patient.

Options for mechanical stabilization and correction of DHS include removable soft or hard collars, Somi brace, halo-vest, or surgical fixation [1-3]. External orthotic devices may provide stabilization and partially correct the deformity; however, they may be cumbersome, irritating the skin, and may cause further deconditioning and deterioration of neck extensor musculature. Therefore these devices are often only temporary solutions, and are likely to achieve long-term satisfaction in only a select patient group-those too old or unwell to undergo major surgical intervention.
At present, the only definitive solution for correction and stabilization of DHS is surgical intervention involving multilevel instrumented fixation and fusion.

Several articles have documented surgical correction of DHS. Dating back to 1988, Simmons and Bradley described a case series of six patients who underwent surgical intervention for what they termed "chin-on-chest deformity" [3]. They advocated halo traction and anterior muscle release to help reverse anterior muscle contraction when the condition was longstanding. They also suggested that posterior resection of the inferior facets could be useful to achieve restoration of normal extension. In four cases, posterior instrumented spinal fusion was performed from mid-cervical (C3-C4) to upper thoracic (T1-T3) levels with good bony fusion, but the authors did not provide longterm follow-up data. They did report that two of these procedures had caused anterior wedge separation, requiring subsequent keystone strut-grafting.

Gerling and Bohlman [4] reported nine cases of DHS in the context of cervical myopathy that were managed with posterior instrumented fusion. The primary complaint of all nine patients was loss of horizontal gaze, and five patients also complained of axial neck pain. Only one was myelopathic, and this patient was not decompressed during surgery. The surgical constructs for all patients spanned C2 to upper thoracic levels (T1-T5) [4, 5]. Four also required anterior cervical releases. The average follow-up was 6 years, and overall the authors found good outcomes at the 2-year follow-up in seven patients and fair outcomes in the other two. This was despite frequent complications including construct failure in one case; two revision surgeries in another; camptocormia in two cases; dysphagia in two cases; and pneumonia in two cases.

Petheram et al [1] described a series of seven patients with DHS, six of whom were treated nonoperatively and one with a C4 to T2 instrumented fusion at another institution. The 79-year-old patient was unhappy with the outcome because of decreased neck mobility and difficulty walking after surgery. The six patients treated nonoperatively also did not fare well, with only one showing marked improvement with physiotherapy and temporary use of a neck collar.

Amin et al presented a patient with long-standing DHS and osteoporosis who was managed with instrumented fusion spanning C2-T11 after suffering a fall and mul- 
tiple thoracic vertebral fractures [6]. Their rationale for the lengthy construct was due to extremely poor-quality bone and the need for stabilization of the thoracic fractures. The patient recovered well and after 3 months of rehabilitation was able to live independently, walking without aids, and maintaining a functionally useful head position.

Kawaguchi et al reported a case of DHS caused solely by cervical myelopathy secondary to spondylosis [7]. In this scenario, they found that decompressive laminectomy alone was sufficient to improve neck extensor function to allow return to normal posture.

A final case describes a patient with Parkinson disease who had undergone DBS placement but continued to have falls and sustained an odontoid fracture. Initial surgical intervention consisted of $\mathrm{Cl}-\mathrm{C} 2$ internal fixation, but the patient sustained a vertebral artery injury and continued to experience painful spasmodic anterocollis and torticollis. This patient subsequently received occiput to $\mathrm{Tl}$ posterior instrumented fusion, which resulted in complete resolution of neck pain and a subjective improvement in overall functioning [8].

We need to consider several key issues in the surgical management of DHS. First, the decision to operate is based on numerous patient factors, including age, functional status, and current quality of life reported by the patient. This has to be weighed against the expected functional status and quality of life after intervention, which will be affected if an occipito-cervico-thoracic fusion is performed.

Second, is the number of levels that need to be stabilized. While most published reports of instrumented fusion spared the craniocervical junction, our construct did not due to the patient's extremely poor extensor musculature. In general, if function of moderate neck extensor remains, it is preferable to limit the rostral level of fusion to C2 to preserve some head mobility. It is also important to consider the status of the thoracic extensor musculature and the bone quality to determine how caudal the fusion needs to be extended. In our case due to poor-density bone the integrity of a shorter construct would have been questionable. In other individuals with high-quality bone and relatively normal thoracic extensor muscles, fusion down to T3 would likely be sufficient.

Third, is the question whether or not to perform a laminectomy in the context of mild cervical myelopathy. It is possible that the mechanical correction of the severe kyphosis itself may decompress the spinal cord. In our case, laminectomy was indicated as the patient had clinical and radiological signs of myelopathy. Given the presence of canal stenosis and cord signal change, the safest method to protect the spinal cord during the correction of deformity was to ensure that it was decompressed first.

Finally, the timing of surgery is vital in this degenerative condition. While it is desirable to delay surgery until it is absolutely necessary, it is essential to intervene before cervical myelopathy and neurological deficits become established. The recommended approach is to monitor closely for clinical or radiological evidence (via serial MRIs) of cervical myelopathy, and intervene if this becomes apparent.

In summary, we report a case of DHS secondary to cervical myopathy. Such a presentation requires a persistent medical workup and treatment of reversible causes before surgical intervention is contemplated. Surgical issues to consider include the optimal timing of intervention, the need for anterior muscle release, the choice of approach (anterior vs posterior vs 360), and the levels which will involve decompression and instrumented fusion. 


\section{REFERENCES}

1. Petheram TG, Hourigan PG, Emran IM, et al (2008) Dropped head syndrome: a case series and literature review. Spine; 33(1):47-51.

2. Gourie-Devi M, Nalini A, Sandhya S (2003) Early or late appearance of 'dropped head syndrome' in amyotrophic lateral sclerosis. J Neurol Neurosurg Psychiatry; 74:683-686.

3. Simmons EH, Bradley DD (1988) Neuro-myopathic flexion deformities of the cervical spine. Spine; 13(7):756-762.

4. Gerling MC, Bohlman HH (2008) Dropped head deformity due to cervical myopathy: surgical treatment outcomes and complications spanning twenty years. Spine; 33(20) :E739-E745.

5. Katirji B, Hachwi R, Al-Shekhlee A, et al (2005) Isolated dropped head due to adultonset nemaline myopathy treated by posterior fusion. Neurology; 65(9):1504-1505.

6. Amin A, Casey AT, Etherington G (2004) Is there a role for surgery in the management of dropped head syndrome? Br J Neurosurg; 18(3):289-293.

7. Kawaguchi A, Miyamoto K, Sakaguchi Y, et al (2004) Dropped head syndrome associated with cervical spondylotic myelopathy. J Spinal Disorder Tech; 17(6):531-534.

8. Pereira EA, Wilson-MacDonald J, Green AL, et al (2010) Posterior occipitocervical instrumented fusion for dropped head syndrome after deep brain stimulation. J Clin Neurosci; 17(4):541-542.

9. Ahdab $R$, Creange $A$, Benaderette $S$, et al (2009) Cervical spondylotic amyotrophy presenting as dropped head syndrome. Clin $\mathrm{Neu}$ rol Neurosurg; 111(10):874-876.

10. Gourie-Devi M, Nalini A, Sandhya S (2003) Early or late appearance of "dropped head syndrome" in amyotrophic lateral sclerosis. J Neurol Neurosurg Psychiatr; 74(5):683-686.

11. Kashihara K, Ohno M, Tomita S (2006) Dropped head syndrome in Parkinson's disease. Mov Disord; 21(8):1213-1216.

12. Umapathi T, Chaudhry V, Cornblath D, et al (2002) Head drop and camptocormia. J Neurol Neurosurg Pyschiatry; 73(1):1-7.

13. Oyama G, Hayashi A, Mizuno Y, et al (2009) Mechanism and treatment of dropped head syndrome associated with parkinsonism. Parkinsonism Rel Disord; 15(3):181-186.

14. Brumback RA, Borge AF, Leech RW (1980) Postpoliomyelitis amyotrophy with rod (nemaline) bodies in skeletal muscle. Trans Am Neurol Assoc; 105:430-431.

15. Hoffman D, Gutmann L (1994) The dropped head syndrome with chronic inflammatory demyelinating polyneuropathy. Muscle Nerve; 17(7):808-810.
16. Maeda K, Ohsaki, Kuki K, et al (1998) Severe antecollis during antipsychotics treatment: a report of three cases. Prog Neuropsychopharmacol Biol Psychiatry; 22(5):749-759.

17. D'Amelio M, Di Benedetto N, Ragonese P, et al (2007) Dropped head as an unusual presenting sign of myasthenia gravis. Neurol Sci; 28(2):104-106.

18. Ueda T, Kanda F, Kobessho H, et al (2009) "Dropped head syndrome" caused by Lambert-Eaton myasthenic syndrome. Muscle Nerve; 40(1):134-136.

19. Kastrup A, Gdynia HJ, Nägele T, et al (2008) Dropped-head syndrome due to steroid responsive focal myositis: a case report and review of the literature. J Neurol Sci; 267:162-165.

20. Garcin B, Lenglet T, Dubourg O, et al (2010) Dropped head syndrome as a presenting sign of scleromyositis. J Neurol Sci; 292:101-103.

21. Kataoka H, Sugie $K$, Terashima $M$, et al (2009) Isolated inflammatory myopathy with rimmed vacuoles presenting with dropped head. Neuromuscul Disord; 19(12):853-855.

22. Katz JS, Wolfe GI, Burns DK, et al (1996) Isolated neck extensor myopathy: a common cause of dropped head syndrome. Neurology; 46(6):917-921.

23. Baquis GD, Moral L, Sorrell M, et al (1997) Neck extensor myopathy: a mitochondrial disease. Neurology; 48:A443.

24. Furby A, Behin A, Lefaucheur JP, et al (2010) Late-onset cervicoscapular muscle atrophy and weakness after radiotherapy for Hodgkin disease: a case series. J Neurol Neurosurg Psychiatr; 81(1):101-104.

25. Rose MR, Levin KH, Griggs RC (1999) The dropped head plus syndrome: quantitation of response to corticosteroids. Muscle Nerve; 22(1):115-118.

26. Karpati G, Carpenter S, Engel AG (1975) The syndrome of systemic carnitine deficiency: clinical, morphologic, biochemical, and pathophysiologic features. Neurology; 25(1):16-24.

27. Askmark H, Olsson Y, Rossitti S (2000) Treatable dropped head syndrome in hypothyroidism. Neurology; 55(6):896-897.

28. Kendall-Taylor P, Turnbull DM (1983) Endocrine myopathies. BMJ (Clin Res Ed); 287(6394):705708.

29. Beekman R, Tijssen CC, Visser LH, et al (2002) Dropped head as the presenting symptom of primary hyperparathyroidism. J Neurol; 249(12):1738-1739.

30. Ryanowski JV, Twydell PT (2009) Treatable dropped head syndrome in hyperparathyroidism. Muscle Nerve; 39(3):409-410.

31. Lerman VJ (1992) Dropped head syndrome. Neurology; 43(4):846. 


\section{COMMENTARY}

Author Michael J Lee

Institution University of Washington, Seattle, WA, USA

Martin and colleagues present a case report and review of the literature surrounding cervical myopathy and resultant dropped head syndrome. They note that this relatively uncommon condition may stem from numerous neuromuscular etiologies. Nonoperative management is largely targeted to the underlying condition and thus may be wide-ranging in its treatment.

This study reveals the paucity of quality literature on this relatively uncommon condition. The largest series describes only nine patients. Consequently, there is no formal standard of care for this condition. Nevertheless, the authors demonstrated appropriate surgical treatment of this patient by adhering to the core tenets of surgical spine treatment: (1) decompression of neural elements, if necessary; (2) stabilization; and (3) correction of deformity.

As Martin and colleagues have properly reviewed, there are other surgical aspects that may provoke additional discussion: approach (anterior vs posterior vs 360), number of levels fused and instrumented, and preemptive decompression. With such a dearth of quality literature, these aspects are best determined on a case-by-case basis. They recommend intervention before onset of cervical myelopathy. In addition, it may be worthwhile to consider earlier intervention while the deformity is passively correctable. Correction of a fixed deformity can often necessitate an anterior and posterior approach, whereas the flexible deformity may be adequately treated with a single approach; thus obviating further surgical insult.

To conclude, the authors presented a well-written case report with an exhaustive literature review on this topic. Although a specific standard of care for this condition has not been defined, adherence to basic core principles of surgical spine treatment, as they have shown, can result in a restoration of function and subsequent improvement in clinical outcome.

\section{EDITORIAL STAFF PERSPECTIVE}

The article on "Dropped head syndrome" (DHS) exemplifies the value of case reports and series. Very rare conditions, such as the one described here, are not really amenable to comparative studies or prospective protocols; they are simply too infrequent and are permeated by high degree of variability. Regarding the general subject of camptocormia (of which the dropped head syndrome is a subset specific to the neck), there is far too little known. There has been no systematic review on this highly disabling and perplexing condition since it was originally described by Alexandre-Achille Souques (1860-1944, Hospice de la Salpêtrière, Paris, France) in 1914 [1-2]. On a historical note, Souques was the cofounder of the Sociéte de Neurologie de Paris together with Ivan Babinski.

One productive outcome of gathering case reports of rare conditions, eg DHS, is the potential to develop more consistent differential diagnosis tables for pathological forward curvature of the spine (with neck and thoracolumbar subsets); and emanating from that, the presentation of sensible diagnostic algorithms to aid clinicians in providing meaningful and systematic assessment approaches.

The EBSJ editorial staff encourages like-minded members of the global spine practitioner community, such as AOSpine, to collaborate on such an endeavor.

1. Azher SN, Jankovic J (2005) Camptocormia: pathogenesis, classification, and response to therapy. Neurology; 65:355359.

2. Souques A, Rodsanoff-Saloff (1914) [La camptocormie; incurvation du tronc, consécutive aux traumatismes du dos et des lombes; considération morphologiques.] Rev Neurol; 28:937-939. French. 